\title{
SOME INTERTWINING RELATIONS MODULO OPERATOR IDEALS
}

\author{
FUAD KITTANEH \\ Department of Mathematics, University of Jordan, Amman, Jordan \\ e-mail:fkitt@ju.edu.jo
}

(Received 2 June, 2005; accepted 12 November, 2005)

\begin{abstract}
Let $B(H)$ denote the algebra of all bounded linear operators on a separable, infinite-dimensional, complex Hilbert space $H$. Let $I$ be a two-sided ideal in $B(H)$. For operators $A, B$ and $X \in B(H)$, we say that Xintertwines $A$ and $B$ modulo $I$ if $A X-X B \in I$. It is easy to see that if $X$ intertwines $A$ and $B$ modulo $I$, then it intertwines $A^{n}$ and $B^{n}$ modulo $I$ for every integer $n>1$. However, the converse is not true. In this paper, sufficient conditions on the operators $A$ and $B$ are given so that any operator $X$ which intertwines certain powers of $A$ and $B$ modulo $I$ also intertwines $A$ and $B$ modulo $J$ for some two-sided ideal $J \supseteq I$.
\end{abstract}

2000 Mathematics Subject Classification. 47A53, 47B10, 47B15, 47B20, 47B47.

1. Introduction. Let $H$ be a separable infinite-dimensional complex Hilbert space. Let $B(H) \supset K(H) \supset F(H)$ denote, respectively, the algebra of all bounded linear operators, the two-sided ideal of compact operators, and the two-sided ideal of finite rank operators on $H$. For any compact operator $T$, let $s_{1}(T), s_{2}(T) \ldots$ be the eigenvalues of $|T|=\left(T^{*} T\right)^{1 / 2}$, arranged in decreasing order and repeated according to multiplicity. A compact operator $T$ is said to be in the Schatten $p$-class $C_{p}(0<p<\infty)$ if $\Sigma_{i} s_{i}(T)^{p}<\infty$. For $p \geq 1$, the Schatten $p$-norm of $T$ is defined by $\|T\|_{p}=\left(\Sigma_{i} s_{i}(T)^{p}\right)^{1 / p}$. This norm makes $C_{p}$ into a Banach space. For all $p>0, C_{p}$ is a two-sided ideal in $B(H)$. Hence, $C_{1}$ is the trace class and $C_{2}$ is the Hilbert-Schmidt class. It is reasonable to let $C_{\infty}$ denote the ideal of compact operators $K(H)$ and $\|\cdot\|_{\infty}$ stand for the usual operator norm. We refer to [7] for the general theory of the Schatten $p$-classes.

Throughout this paper, every ideal in $B(H)$ is assumed to be two-sided and proper. It is well known that if $I$ is a non-trivial ideal in $B(H)$, then $F(H) \subseteq I \subseteq C_{\infty}$.

Let $I$ be an ideal in $B(H)$; let $A, B$ and $X \in B(H)$. We say that $X$ intertwines $A$ and $B$ modulo $I$ if $A X-X B \in I$. If $X$ intertwines $A$ and $B$ modulo the trivial ideal, i.e., if $A X-X B=0$, then we simply say that $X$ intertwines $A$ and $B$. It is easy to see that if $X$ intertwines $A$ and $B$ modulo $I$, then it intertwines $A^{n}$ and $B^{n}$ modulo $I$ for every integer $n>1$. Of course, the converse is not true. Consider, for example, the case in which $A$ and $B$ are non-zero nilpotent operators.

It is the object of this paper to present some sufficient conditions on the operators $A$ and $B$ so that any operator $X$ which intertwines certain powers of $A$ and $B$ modulo $I$, also intertwines $A$ and $B$ modulo $J$, where $J$ is an ideal in $B(H)$ such that $I \subseteq J$. Our results generalize earlier results on this problem by Al-Moajil [1], Duggal [5], and the author [10]. 
2. Intertwining relations modulo arbitrary ideals. In [8], extending a result of Al-Moajil [1], the author has proved the following results.

Theorem A. Let $A, B$ and $X \in B(H)$, where $A$ and $B^{*}$ are subnormal. If $A^{2} X=X B^{2}$ and $A^{3} X=X B^{3}$, then $A X=X B$.

Theorem B. Let $A, B$ and $X \in B(H)$, where $A$ and $B^{*}$ are subnormal. If $A^{2} X-$ $X B^{2} \in F(H)$ and $A^{3} X-X B^{3} \in F(H)$, then $A X-X B \in F(H)$.

Theorem C. Let $A, B$ and $X \in B(H)$, where $A$ and $B^{*}$ are subnormal. If $A^{2} X-$ $X B^{2} \in C_{p}$ and $A^{3} X-X B^{3} \in C_{p}$, for some $p$ with $1 \leq p \leq \infty$, then $A X-X B \in C_{8 p}$.

By a slight modification of the proof of Theorem $C$ given in [10], Duggal [5, Theorem 5] has extended this result to relatively prime powers other than 2 and 3.

The purpose of this section is to extend these results to larger classes of operators and to relatively prime powers other than 2 and 3 . In fact, these theorems follow as immediate consequences of a general result (Theorem 1), which is valid for arbitrary ideals in $B(H)$.

The following lemma, which is in the spirit of Lemma 1.1 in [1], indicates that in order to generalize Theorems A, B and C cited above, it is sufficient to consider two consecutive powers rather than two relatively prime powers.

Lemma 1. Let $I$ be an ideal in $B(H)$. If $A, B$ and $X \in B(H)$ are such that $A^{m} X-$ $X B^{m} \in I$ and $A^{n} X-X B^{n} \in I$, for some relatively prime positive integers $m$ and $n$, then $A^{k} X-X B^{k} \in I$ and $A^{k+l} X-X B^{k+1} \in I$, for some integer $k>1$.

Proof. Since $m$ and $n$ are relatively prime positive integers, there exist integers $s$ and $t$ such that $s m+t n=1$ and $s t$ is negative; say $s$ is negative and $t$ is positive. The assumptions $A^{m} X-X B^{m} \in I$ and $A^{n} X-X B^{n} \in I$ imply that $A^{-m s} X-X B^{-m s} \in I$ and $A^{n t} X-X B^{n t} \in I$. Since $n t=-m s+1$, the result now follows by letting $k=-m s$.

The following elegant factorization result, which is due to Douglas [4], will be essential for us to accomplish our goal.

LEMma 2. Let $T, S \in B(H)$. Then the following conditions are equivalent:

(a) $\operatorname{ran} T \subseteq \operatorname{ran} S$, where ran $T$ denotes the range of $T$;

(b) $T T^{*} \leq c S S^{*}$, for some constant $c>0$;

(c) $T=S R$, for some $R \in B(H)$.

It follows immediately from Lemma 2 that if $T \in B(H)$ is hyponormal, i.e., if $T T^{*} \leq T^{*} T$, then $\operatorname{ran} T \subseteq \operatorname{ran} T^{*}$. Also, if $\operatorname{ran} T \subseteq \operatorname{ran} T^{*}$, then $T=T^{*} R$ for some $R \in B(H)$, and hence $T^{*}=R^{*} T$. Thus, if $\operatorname{ran} T \subseteq \operatorname{ran} T^{*}$, if $X \in B(H)$, and if $I$ is an ideal in $B(H)$, then $T X \in I$ implies $T^{*} X \in I$. The particular case, that hyponormal operators have this property, has been observed by Weiss in [12].

If $I$ and $J$ are ideals in $B(H)$, let $I . J$ denote the ideal generated by products of the form $T S$ with $T \in I$ and $S \in J$. Hence, by induction, $I^{n}$ is defined as $I^{n}=I^{n-1}$. $I$ for every integer $n>1$. It is well known (using the polar decomposition) that every ideal $I$ in $B(H)$ is self-adjoint; i.e., $T \in I$ if and only if $T^{*} \in I$. Also, $|T|^{2} \in I^{2}$ if and only if $|T| \in I$, and $|T| \in I$ if and only if $T \in I$. Consequently, $T^{*} T \in I$ if and only if $T \in I^{1 / 2}$, where $I^{1 / 2}$ is the unique ideal whose square is $I$. For any integer $n>1, I^{1 / n}$ is defined in the obvious way.

We are now in a position to prove the main result of this section. 
Theorem 1. Let $I$ be an ideal in $B(H)$. Let $A, B$ and $X \in B(H)$ with $\operatorname{ran} A \subseteq \operatorname{ran}$ $A^{*}$ and $\operatorname{ran} B^{*} \subseteq \operatorname{ran} B$. If $A^{n} X-X B^{n} \in I$ and $A^{n+1} X-X B^{n+1} \in I$, for some integer $n>1$, then $A X-X B \in I^{1 / 2^{n+1}}$.

Proof. Let $C=A X-X B$. Then simple algebra shows that $A^{n} C \in I$ and $C B^{n} \in I$. Since $\operatorname{ran} A \subseteq \operatorname{ran} A^{*}$, and since $A^{n} C \in I$, it follows that $A^{*} A^{n-1} C \in I$. Thus, $\left(A^{n-1} C\right)^{*}\left(A^{n-1} C\right) \in I$, and so $A^{n-1} C \in I^{1 / 2}$. Continuing down in this way, we obtain that $A C \in I^{1 / 2^{n-1}}$. Again, the assumption $\operatorname{ran} A \subseteq$ ran $A^{*}$ implies that $A^{*} C \in$ $I^{1 / 2^{n-1}}$. Similarly, since $\operatorname{ran} B^{*} \subseteq$ ran $\mathrm{B}$, and since $B^{* n} C^{*}=\left(C B^{n}\right)^{*} \in I$, it follows that $B^{*} C^{*} \in I^{1 / 2^{n-1}}$. Hence, $B C^{*} \in I^{1 / 2^{n-1}}$, and so $C B^{*} \in I^{1 / 2^{n-1}}$. But then $C C^{*} C=$ $C\left(X^{*} A^{*}-B^{*} X^{*}\right) C=C X^{*} A^{*} C-C B^{*} X^{*} C \in I^{1 / 2^{n-1}}$. Hence, $\left(C^{*} C\right)^{2} \in I^{1 / 2^{n-1}}$, which implies that $C^{*} C \in I^{1 / 2^{n}}$, and so $C \in I^{1 / 2^{n+1}}$. This completes the proof.

An important special case of the range inclusion requirement in Theorem 1 is that $A$ and $B^{*}$ are hyponormal operators (in particular subnormal operators). The most interesting ideals for which Theorem 1 is applied are $\{0\}, F(H)$ and $C_{p}(0<p \leq \infty)$. Hence, Theorems A, B and C can be obtained as corollaries of Theorem 1 upon considering the following cases:

(a) $I=\{0\}$, and so $I^{1 / 2^{n+1}}=\{0\}$,

(b) $I=F(H)$, and so $I^{1 / 2^{n+1}}=F(H)$,

(c) $I=C_{p}$, and so $I^{1 / 2^{n+1}}=C_{2^{n+1} p}(0<p \leq \infty)$.

Here we have, respectively, used the facts that $T=0$ if and only $T^{*} T=0, T \in F(H)$ if and only if $T^{*} T \in F(H)$, and $T \in C_{2 p}$ if and only if $T^{*} T \in C_{p}(0<p \leq \infty)$.

We should like to close this section by remarking that if $I=\{0\}$ then, in Theorem 1 , the conditions that $\operatorname{ran} A \subseteq \operatorname{ran} A^{*}$ and $\operatorname{ran} B^{*} \subseteq \operatorname{ran} B$ can be weakened so that $\overline{\text { ran }}$ $A \subseteq \overline{\operatorname{ran}} A^{*}$ and $\overline{\operatorname{ran}} B^{*} \subseteq \overline{\operatorname{ran}} B$, or equivalently, (by taking orthogonal complements) $\operatorname{ker} A \subseteq \operatorname{ker} A^{*}$ and ker $B^{*} \subseteq \operatorname{ker} B$, where $\operatorname{ker} A$ and $\overline{\operatorname{ran}} A$ denote the kernel of $A$ and the closure (in the usual Hilbert space topology) of $\operatorname{ran} A$, respectively. However, these conditions cannot be replaced by the symmetric conditions that $\operatorname{ran} A \subseteq \operatorname{ran} A^{*}$ and ran $B \subseteq \operatorname{ran} B^{*}$, or even more strongly, that $A$ and $B$ are hyponormal operators. This may be concluded from Remark 3.2 (a) in [1] or by considering the following example.

EXAMPLE 1. Let $\left\{e_{n}\right\}_{n=1}^{\infty}$ be an orthonormal basis for $H$. Let $U$ be the unilateral shift operator defined by $U e_{n}=e_{n+1}$, for all $n$, and let $P$ be the orthogonal projection on the subspace spanned by $e_{1}$ and $e_{2}$; i.e., $P e_{1}=e_{1}, P e_{2}=e_{2}$ and $P e_{n}=0$ for $n>2$. On $H \oplus H$, let $T=\left[\begin{array}{cc}0 & 0 \\ 0 & U\end{array}\right]$ and $X=\left[\begin{array}{ll}0 & p \\ 0 & 0\end{array}\right]$. Then $T$ is hyponormal, $T^{2} X=X T^{2}$ and $T^{3} X=$ $X T^{3}$, but $T X \neq X T$. In fact, every product involved here is zero except $X T=\left[\begin{array}{cc}0 & p U \\ 0 & 0\end{array}\right]$, which is non-zero because $P U e_{1}=e_{2}$.

3. Intertwining relations modulo non-trivial ideals. In [5], Duggal has proved the following two results.

Theorem D. Let $A, B$ and $X \in B(H)$ such that $A$ is semi-Fredholm with ind $A \leq 0$ or $B$ is semi-Fredholm with ind $B \geq 0$. If $A^{m} X-X B^{m} \in C_{p}$ and $A^{n} X-X B^{n} \in C_{p}$, for some relatively prime positive integers $m$ and $n$, and some $p$ with $1 \leq p \leq \infty$, then $A X-$ $X B \in C_{p}$.

Theorem E. Let $A, B$ and $X \in B(H)$ such that $1-A^{*} A \in C_{p}$ or $1-B^{*} B \in C_{p}$, for some $p$ with $1 \leq p \leq \infty$. If $A^{m} X-X B^{m} \in C_{p}$ and $A^{n} X-X B^{n} \in C_{p}$, for some relatively prime positive integers $m$ and $n$, then $A X-X B \in C_{p}$. 
Theorem $\mathrm{D}$ is a generalization of Theorem 7 in [10]. It should be mentioned here that the condition $1-B^{*} B \in C_{p}$ in Theorem $\mathrm{E}$ should be replaced by $1-B B^{*} \in C_{p}$. To see this, let $H^{(\infty)}=\oplus_{n=1}^{\infty} H_{n}$, where $H_{n}=H$ for all $n$, and let $B$ be the operator valued weighted shift

$$
B=\left[\begin{array}{ccccc}
0 & 0 & 0 & 0 & \cdots \\
1 & 0 & 0 & 0 & \cdots \\
0 & 1 & 0 & 0 & \cdots \\
0 & 0 & 1 & 0 & \cdots \\
\vdots & \vdots & \ddots & \ddots & \ddots
\end{array}\right]
$$

$A=B^{*}$ and

$$
X=\left[\begin{array}{ccccc}
1 & 0 & 0 & 0 & \cdots \\
0 & 1 & 0 & 0 & \cdots \\
0 & 0 & 0 & 0 & \cdots \\
0 & 0 & 0 & 0 & \cdots \\
\vdots & \vdots & \vdots & \vdots & \vdots
\end{array}\right]
$$

Then $1-A A^{*}=1-B^{*} B=0 \in C_{p}$ for all $p$ with $0<p \leq \infty$, and

$$
1-A^{*} A=1-B B^{*}=\left[\begin{array}{ccccc}
1 & 0 & 0 & 0 & \ldots \\
0 & 0 & 0 & 0 & \cdots \\
0 & 0 & 0 & 0 & \cdots \\
0 & 0 & 0 & 0 & \cdots \\
\vdots & \vdots & \vdots & \vdots & \vdots
\end{array}\right] \notin C_{p}
$$

for all $p$ with $0<p \leq \infty$. Moreover, $A^{2} X=X B^{2}=A^{3} X=X B^{3}=0$ and

$$
A X-X B=\left[\begin{array}{ccccc}
0 & 1 & 0 & 0 & \cdots \\
-1 & 0 & 0 & 0 & \cdots \\
0 & 0 & 0 & 0 & \cdots \\
0 & 0 & 0 & 0 & \cdots \\
\vdots & \vdots & \vdots & \vdots & \vdots
\end{array}\right] \notin C_{p}
$$

for all $p$ with $0<p \leq \infty$.

In this section we refine these results by extending them in two directions: to larger classes of operators and to all non-trivial ideals in $B(H)$.

Note that $A \in B(H)$ is a semi-Fredholm operator if ran $A$ is closed and either ker $A$ or ker $A^{*}$ is finite-dimensional. It is well known that ran $A$ is closed if and only if ran $A^{*}$ is closed. Thus, $A$ is a semi-Fredholm operator if and only if $A^{*}$ is semi-Fredholm. The index of a semi-Fredholm operator $A$ is given by ind $A=\operatorname{dim} \operatorname{ker} A-\operatorname{dim} \operatorname{ker} A^{*}$. Hence, ind $A^{*}=-$ ind $A$. A semi-Fredholm operator $A$ is a Fredholm operator if $-\infty<$ ind $A<\infty$; i.e., $A$ is a Fredholm operator if $\operatorname{ran} A$ is closed and both ker $A$ and $\operatorname{ker} A^{*}$ are finite-dimensional. An operator $A \in B(H)$ is said to be left invertible modulo an ideal $I$ in $B(H)$, if there exists an operator $B \in B(H)$ such that $1-B A \in I$; i.e., the coset $v(A)$ is left invertible in the quotient algebra $B(H) / I$, where $v$ is the canonical homomorphism of $B(H)$ onto $B(H) / I$. The (two-sided) invertibility modulo $I$ is defined in the obvious way. It has been shown in [6, Theorem 1.1] that for $A \in B(H)$, 
$\operatorname{ran} A$ is closed and ker $A$ is finite-dimensional if and only if $A$ is left invertible modulo $C_{\infty}$. The following lemma asserts that in this characterization $C_{\infty}$ can be replaced by any non-trivial ideal in $B(H)$.

Lemma 3. Let I be a non-trivial ideal in $B(H)$. Then for $A \in B(H)$, ran $A$ is closed and ker $A$ is finite-dimensional if and only if $A$ is left invertible modulo $I$.

Proof. Since $I \subseteq C_{\infty}$, the "if" part follows by Theorem 1.1 in [6]. Now we prove the "only if" part. Assume that $\operatorname{ran} A$ is closed and $\operatorname{ker} A$ is finite-dimensional. Then ind $A \neq \infty$. If ind $A$ is finite, then $A$ is a Fredholm operator. Hence, by Atkinson's theorem [8, p. 96], $A$ is invertible modulo $F(H)$. Since $I$ is non-trivial, and hence $F(H) \subseteq I$, it follows that $A$ is invertible modulo $I$. If, on the other hand, ind $A=-\infty$, then it follows from Proposition XI.3.21 in [3] that there exists a finite rank operator $F \in F(H)$ such that $T=A+F$ is left invertible. Let $S \in B(H)$ be a left inverse of $T$. Then $S A+S F=1$, and so $1-S A \in F(H)$. Consequently, $1-S A \in I$. Thus, in either case $A$ is left invertible modulo $I$ and the proof is complete.

One version of our main result of this section can be stated as follows.

THEOREM 2. Let I be a non-trivial ideal in $B(H)$. Let $A, B$ and $X \in B(H)$, where either $\operatorname{ran} A$ is closed and $\operatorname{ker} A$ is finite-dimensional or $\operatorname{ran} B$ is closed and $\operatorname{ker} B^{*}$ is finite-dimensional. If $A^{n} X-X B^{n} \in I$ and $A^{n+l} X-X B^{n+l} \in I$, for some integer $n>1$, then $A X-X B \in I$.

Proof. As in the proof of Theorem 1, let $C=A X-X B$. Then $A^{n} C \in I$ and $C B^{n} \in I$. If $\operatorname{ran} A$ is closed and ker $A$ is finite-dimensional then, by Lemma 3, $A$ is left invertible modulo $I$, and so there exists an operator $S \in B(H)$ such that $1-S A \in I$. But then $1-S^{n} A^{n} \in I$. This, together with $A^{n} C \in I$, implies that $C \in I$. On the other hand, if $\operatorname{ran} B$ is closed (and hence $\operatorname{ran} B^{*}$ is closed) and $\operatorname{ker} B^{*}$ is finite-dimensional then, by Lemma $3, B^{*}$ is left invertible modulo $I$. In view of this, $B^{* n} C^{*}=\left(C B^{n}\right)^{*} \in I$ now implies that $C^{*} \in I$. Hence, $C \in I$, and the proof is complete.

In terms of the index function, the hypotheses on $A$ and $B$ in Theorem 2 can be restated so that either $A$ is semi-Fredholm with ind $A \neq \infty$ or $B$ is semi-Fredholm with ind $B \neq-\infty$. Now, in view of Lemma 3, Theorem D and the corrected version of Theorem $\mathrm{E}$ follow as special cases of Theorem 2 .

At the end of this section, we should like to give the following example, which shows that Theorem 2 is not valid for the trivial ideal $I=\{0\}$.

ExAmple 2. Let $U$ and $P$ be the operators defined in Example 1. On $H \oplus H$, let $T=\left[\begin{array}{cc}U & 0 \\ 0 & U^{*}\end{array}\right]$ and $X=\left[\begin{array}{ll}0 & 0 \\ P & 0\end{array}\right]$. Then ran $T$ is closed and both ker $T$ and $\operatorname{ker} T^{*}$ are onedimensional subspaces of $H \oplus H$; i.e., $T$ is a Fredholm operator with ind $T=0$. Since $U^{* 2} P=P U^{2}=0$ and $U^{*} P \neq P U$, simple matrix computations show that $T^{2} X=X T^{2}=0$, and so $T^{3} X=X T^{3}=0$, but $T X \neq X T$.

4. Intertwining relations modulo the trivial ideal. This section is mainly devoted to the case $I=\{0\}$. Let $A \in B(H)$ and let $\sigma(A)$ denote its spectrum. Then, by the Riesz functional calculus, any $X \in B(H)$ that commutes with $A$ also commutes with $f(A)$ for every function $f$ that is analytic on some neighbourhood of $\sigma(A)$ Recall that the operator $f(A)$ is defined by $f(A)=\frac{1}{2 \pi i} \int_{\Gamma} f(z)(z-A)^{-1} d z$, where $\Gamma$ is any Jordan system in the domain of $f$ that contains $\sigma(A)$ in its 'inside'.

For the reader's convenience, a proof of the following "folk" result is included. 
THEOREM 3. Let $A, X \in B(H)$. If $f$ is a function that is one-to-one and analytic on some neighbourhood of $\sigma(A)$, then $f(A) X=X f(A)$ if and only if $A X=X A$.

Proof. We have only to prove the "only if" part. Assume that $f(A) X=X f(A)$ and let $\Omega$ be the domain of analyticity of $f$ such that $\sigma(A) \subset \Omega$. Then $f^{-1}$ is one-to-one and analytic on $f(\Omega)$. By the spectral mapping theorem, $\sigma(f(A))=f(\sigma(A)) \subset f(\Omega)$. Now $f(A) X=X f(A)$ implies that $f^{-1}(f(A)) X=X f^{-1}(f(A))$. But the basic properties of the Riesz functional calculus show that $A=f^{-1}(f(A))$. Hence, $A X=X A$, as required.

An intertwining version of Theorem 3 is now presented.

THEOREM 4. Let $A, B$ and $X \in B(H)$. Iff is a function that is one-to-one and analytic on some neighbourhood of $\sigma(A) \cup \sigma(B)$, then $f(A) X=X f(B)$ if and only if $A X=X B$.

Proof. Define operators $T$ and $Y$ on the Hilbert space $H \oplus H$ by $T=\left[\begin{array}{cc}A & 0 \\ 0 & B\end{array}\right], Y=$ $\left[\begin{array}{cc}0 & X \\ 0 & 0\end{array}\right]$. Then $\sigma(T)=\sigma(A) \cup \sigma(B)$ and $f(T)=\left[\begin{array}{cc}f(A) & 0 \\ 0 & f(B)\end{array}\right]$. Since, by simple algebra, $A X=$ $X B$ if and only if $T Y=Y T$, and $f(A) X=X f(B)$ if and only if $f(T) Y=Y f(T)$, the result now follows by applying Theorem 3 to the operators $T$ and $Y$.

For our purpose, the most interesting cases of Theorem 4 are demonstrated in the following corollaries.

Corollary 1. Let $A, B$ and $X \in B(H)$, where $A$ and $B$ are self-adjoint. Then, for every odd positive integer $n, A^{n} X=X B^{n}$ if and only if $A X=X B$.

The requirement that $n$ is an odd positive integer in Corollary 1 can be dropped, if the condition on $A$ and $B$ is strengthened as follows.

Corollary 2. Let $A, B$ and $X \in B(H)$, where $A$ and $B$ are positive. Then, for every positive real number $r, A^{r} X=X B^{r}$ if and only if $A X=X B$.

Using the simple (but very useful) observation that, for any $A \in B(H)$, the matrix $\left[\begin{array}{cc}0 & A \\ A^{*} & 0\end{array}\right]$ defines a self-adjoint operator on $H \oplus H$, enables us to prove the main result of this section.

THEOREM 5. Let $A, B$ and $X \in B(H)$. Then, for every positive integer $n,\left(A A^{*}\right)^{n} A X=$ $X\left(B B^{*}\right)^{n} B$ and $\left(A^{*} A\right)^{n} A^{*} X=X\left(B^{*} B\right)^{n} B^{*}$ if and only if $A X=X B$ and $A^{*} X=X B^{*}$.

Proof. On $H \oplus H$, let $T=\left[\begin{array}{cc}0 & A \\ A^{*} & 0\end{array}\right], S=\left[\begin{array}{cc}0 & B \\ B^{*} & 0\end{array}\right]$ and $Y=\left[\begin{array}{cc}X & 0 \\ 0 & X\end{array}\right]$. Then $T$ and $S$ are self-adjoint. Simple algebra shows that

$$
T^{2 n+1}=\left[\begin{array}{cc}
0 & \left(A A^{*}\right)^{n} A \\
\left(A^{*} A\right)^{n} A^{*} & 0
\end{array}\right] \quad \text { and } \quad S^{2 n+1}=\left[\begin{array}{cc}
0 & \left(B B^{*}\right)^{n} B \\
\left(B^{*} B\right)^{n} B^{*} & 0
\end{array}\right]
$$

Now the conditions $\left(A A^{*}\right)^{n} A X=X\left(B B^{*}\right)^{n} B$ and $\left(A^{*} A\right)^{n} A^{*} X=X\left(B^{*} B\right)^{n} B^{*}$ are equivalent to saying that $T^{2 n+1} Y=Y S^{2 n+1}$. But this last condition is equivalent, by Corollary 1, to saying that $T Y=Y S$, which is also equivalent to saying that $A X=X B$ and $A^{*} X=X B^{*}$. The proof is now complete.

Corollary 3. Let $A, B$ and $X \in B(H)$, where $A$ and $B$ are normal. Then, for every positive integer $n,\left(A A^{*}\right)^{n} A X=X\left(B B^{*}\right)^{n} B^{*}$ if and only if $A X=X B$.

Proof. We first observe that the adjoint of $\left(A A^{*}\right)^{n} A$ is $\left(A^{*} A\right)^{n} A^{*}$. Now the result follows from Theorem 4 and the Fuglede-Putnam theorem. 
The normality assumption in Corollary 3 is essential, even in the finite-dimensional setting. For example, consider $A=\left[\begin{array}{cc}1 & 1 \\ -1 & 0\end{array}\right]$ and $X=\left[\begin{array}{cc}1 & 1 \\ -1 & -1\end{array}\right]$ acting on a two-dimensional Hilbert space. Then $\left(A A^{*} A\right) X=X\left(A A^{*} A\right)$, but $A X \neq X A$.

If, in Corollary 3, we take $X$ to be the identity operator, then we have the following generalization of a finite-dimensional result of Khatri [9, Theorem 3(iii)].

Corollary 4. Let $A, B \in B(H)$. Then, for every positive integer $n,\left(A A^{*}\right)^{n} A=$ $\left(B B^{*}\right)^{n} B$ if and only if $A=B$. In particular, $\left(A A^{*}\right)^{n} A=\left(A^{*} A\right)^{n} A^{*}$ if and only if $A=A^{*}$; i.e., $\left(A A^{*}\right)^{n} A$ is self-adjoint if and only if $A$ is self-adjoint.

Proof. If $\left(A A^{*}\right)^{n} A=\left(B B^{*}\right)^{n} B$ then, by taking the adjoints of both sides of this equation, we also have $\left(A^{*} A\right)^{n} A^{*}=\left(B^{*} B\right)^{n} B^{*}$. The result now follows from Theorem 5.

We conclude with the following two remarks concerning Section 4.

REMARKS. (1) The intertwining relations in this section can also be taken modulo $C_{\infty}$. Just consider the Calkin algebra $B(H) / C_{\infty}$, which is a $C^{*}$-algebra, and hence it can be represented as an operator algebra.

(2) It follows from Theorem 3 in [2] that if $A, B \in B(H)$ are self-adjoint, and if $X \in B(H)$ is such that $A^{n} X-X B^{n} \in C_{p}$, for some odd positive integer $n$ and some $p$ with $1 \leq p \leq \infty$, then $A X-X B \in C_{n p}$. It also follows from Theorem 7 in [2] that if $A, B \in B(H)$ are positive, and if $X \in B(H)$ is such that $A^{r} X-X B^{r} \in C_{p}$, for some real number $r \geq 1$ and some $p$ with $1 \leq p \leq \infty$, then $A X-X B \in C_{r p}$. Moreover, it follows from Theorem 3.1 in [11] that if either $A$ or $B$ is invertible, then $A X-X B \in C_{p}$.

\section{REFERENCES}

1. A. H. Al-Moajil, The commutants of relatively prime powers in Banach algebras, Proc. Amer. Math. Soc. 57 (1976), 243-249.

2. R. Bhatia and F. Kittaneh, Some inequalities for norms of commutators, SIAM J. Matrix Anal. Appl. 18 (1997), 258-263.

3. J. B. Conway, A course in functional analysis (Springer-Verlag, 1985).

4. R. G. Douglas, On majorization, factorization, and range inclusion of operators on Hilbert space, Proc. Amer. Math. Soc. 17 (1966), 413-415.

5. P. B. Duggal, The commutant modulo $C_{p}$ of co-prime powers of operators on a Hilbert space, J. Math. Anal. Appl. 263 (2001), 110-120.

6. P. A. Fillmore, J. G. Stampfli and J. P. Williams, On the essential numerical range, the essential spectrum, and a problem of Halmos, Acta Sci. Math. (Szeged) 33 (1972), 179-192.

7. I. C. Gohberg and M. G. Krein, Introduction to the theory of linear nonself-adjoint operators, Transl. Math. Monographs, Vol. 18 (Amer. Math. Soc., Providence, R. I., 1969). $57-65$.

8. P. R. Halmos, A Hilbert space problem book (Springer-Verlag, 1982).

9. C. G. Khatri, Powers of matrices and idempotency, Linear Algebra Appl. 33 (1980),

10. F. Kittaneh, On the commutants modulo $C_{p}$ of $A^{2}$ and $A^{3}, J$. Austral. Math. Soc. Ser. A 41 (1986), 47-50.

11. F. Kittaneh and H. Kosaki, Inequalities for the Schatten p-norm V, Publ. RIMS, Kyoto Univ. 23 (1987), 433-443.

12. G. Weiss, The Fuglede commutativity theorem modulo operator ideals, Proc. Amer. Math. Soc. 83 (1981), 113-118. 